\title{
ROBOTIC HUMANOID ARM
}

\section{A.C. GHEORGHE}

Valahia University of Targoviste, Faculty of Electrical Engineering, Electronics and Information Technology, Romania E-mail: gheorghe_andrei89@yahoo.com

\begin{abstract}
The study aims for the development of a robotic humanoid arm for prosthesis, developed at the level of a prototype, with the help of which the user can replace a lost or missing limb. The movement of the fingers is done through servomotors and the control for the prototype is obtained by using a mobile application, this control method is used strictly for testing. The present project has a custom control board that is made from a Arduino Nano development board, Bluetooth HC-05 module, power/servo pins and provides a viable technical interfacing solution for testing the above mentioned equipment, using the Arduino Nano resources and the IDE/Java software resources.
\end{abstract}

Keywords: Arduino, Android, Bluetooth

\section{INTRODUCTION}

An advanced industrialized society supposes a flexible automation of the all processes, in which the robots have a determining role. Since robots are flexible, providing freedom of movement similar to those of the upper limbs of human beings, their use produces a number of economic and social benefits. These include: increased productivity of products and faster recovery of investments. The creation of automation means such as manipulators and robots was determined, among others, by the increase of the wear of the produced parts and by the reduction of the relative share of mass and large series productions due to the production of unique and small series. The flexible automation, representing the highest level of programmable automation, is organized for the discrete production in batches, in manufacturing cells controlled and driven by the computer and serviced by one or more robots. This is how, through the introduction of industrial manipulators and robots, the transformation of production systems from human-machine systems to human-machine robot systems has been introduced. This transformation leads to the release of workers from dangerous or uncomfortable work. The robot used in manufacturing processes is a human substitute, being able to replace, at the current technological level, the functions of the hands, being unable to have legs. The word, robot, has today almost a century of life appearing for the first time in the play R.U.R. (Rossum's Universal Robot), written by a Czech playwriter and used internationally since 1923, when the mentioned work was translated into English. The term robotics was coined by Isaac Asimov, one of the great writers of scientific-fantasy literature. This writer first used the word, "robotics" in 1942, in the Runaround story, in which he establishes from the beginning the three principles of a "robot". These three principles, as stated by Asimov, are: - A robot does not harm human beings and does not inactively witness any danger in which a human being is involved. - A robot must obey the commands given to it by human beings, except those who do not respect the first principle. - A robot must protect itself from injury, unless the first two principles are violated. The design of the robotic arm developed for this study was made in SolidWorks, the robotic arm and the control board are made of this components:

- Arduino Nano development board that has the ATmega328p processor.

- $\quad$ MG996R servomotor, the servo has metal gearing that are used for better torque $\left(10 \mathrm{~kg} / \mathrm{cm}^{2}\right)$.

- Bluetooth HC-05 module that is a Bluetooth SPP (Serial Port Protocol) module.

\subsection{Arduino Nano}

The development board (Figure 1) is equipped with the same high-performance micro-controller (ATmega328p) that we can find on the Arduino Uno and the $\mathrm{CH} 340$ serial USB converter that is used to program the board over USB. Its advantage is represented by its small size, so it can be integrated into various projects where component space is very important. Device programming is done via a cable with the mini USB port and the development board comes with a bootloader. The programing language used for the Nano board is IDE (Integrated Development Environment), the language is easy to understand and use to program the development board. The board has a clock speed of $16 \mathrm{MHz}$, flash memory of $32 \mathrm{~KB}, 6 \mathrm{PWM}$ (Pulse Width Modulation) pins and has the operating voltage of 5 $\mathrm{V}$.

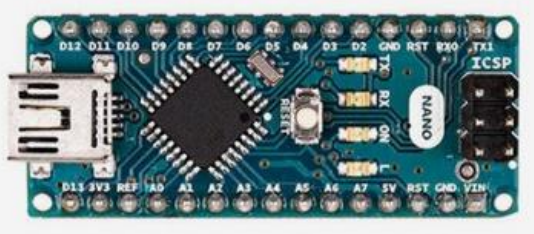

Figure 1. Arduino Nano development board.

\subsection{MG996R Servomotor}

The MG996R servomotor (Figure 2) is small in size (40.7 x 19.7 x $42.9 \mathrm{~mm}$ ) and has a weight of $55 \mathrm{~g}$, the servo has a stall torque of $11 \mathrm{kgf}^{*} \mathrm{~cm}$ (at $6 \mathrm{~V}$ ), operating voltage of $4.8 \mathrm{~V}-7.2 \mathrm{~V}$, stable and shock proof double ball bearing design and can operate in a temperature range of $0-55^{\circ} \mathrm{C}$ 


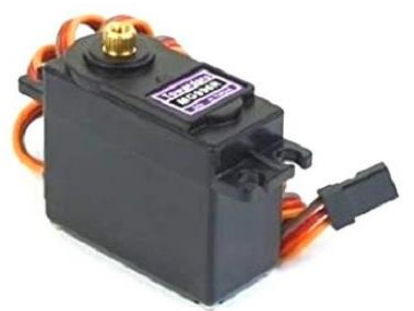

Figure 2. MG996R servomotor.

\subsection{Bluetooth HC-05 module}

The HC-05 Bluetooth module (Figure 3) can be implemented in projects where you want to control different devices with your Android smartphone or laptop via Bluetooth. The user can configure the module as master or slave and switch between the two modes to allow data transmission or reception. The module works on a frequency of $2.4 \mathrm{Ghz}$ ISM band and has the Bluetooth specification v2.0 + EDR, speed of $2.1 \mathrm{Mbps}(\mathrm{Max})$ in asynchronous mode and $1 \mathrm{Mbps}$ in synchronous mode, authentication and encryption security, working temperature of $-20 \sim 75{ }^{\circ} \mathrm{C}$, operating voltage of 5 Vand $26.9 \mathrm{~mm} \times 13 \mathrm{~mm} \times 2.2 \mathrm{~mm}$ dimensions.

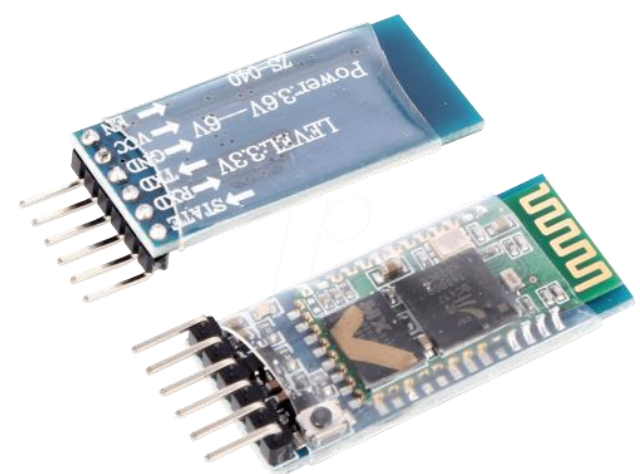

Figure 3. Bluetooth HC-05 module

\section{ROBOTIC HUMANOID HAND}

In Figure 4 we can see the schematic of the control board that was made for testing the motion of the fingers, the board is composed of a Arduino Nano, Bluetooth HC-05 module and connectors for the servomotors.

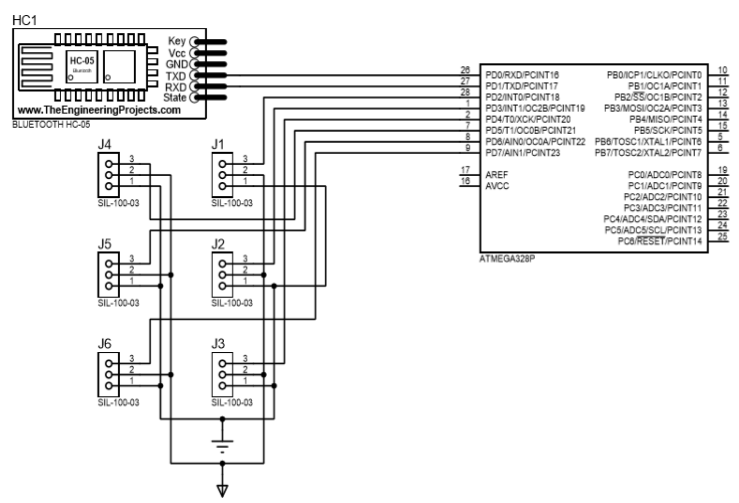

Figure 4. Control board schematic.
In Figure 5 we have the control board fully assembled with all the parts soldered on the prototype board and the power connector for powering the board and servomotors.

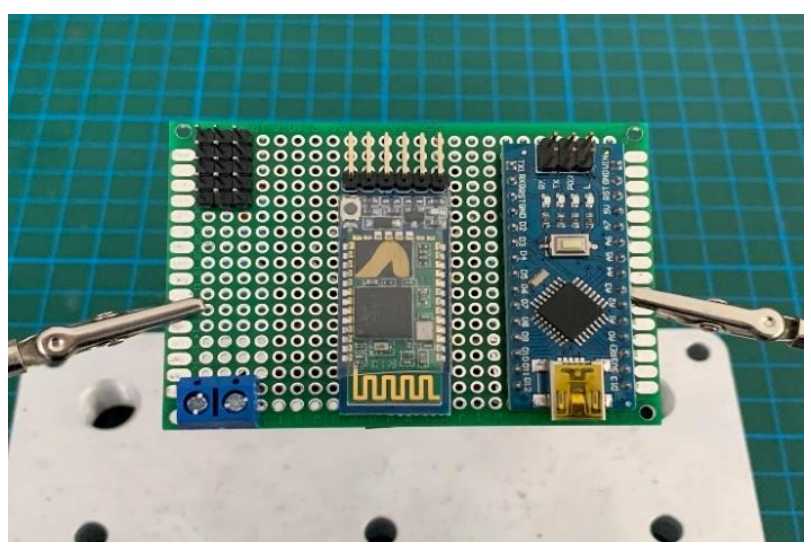

Figure 5. Control board.

In Figure 6 we can see the 3D printed parts for the robotic hand witch include the fingers, palm, forearm, servomotor mount and elbow.

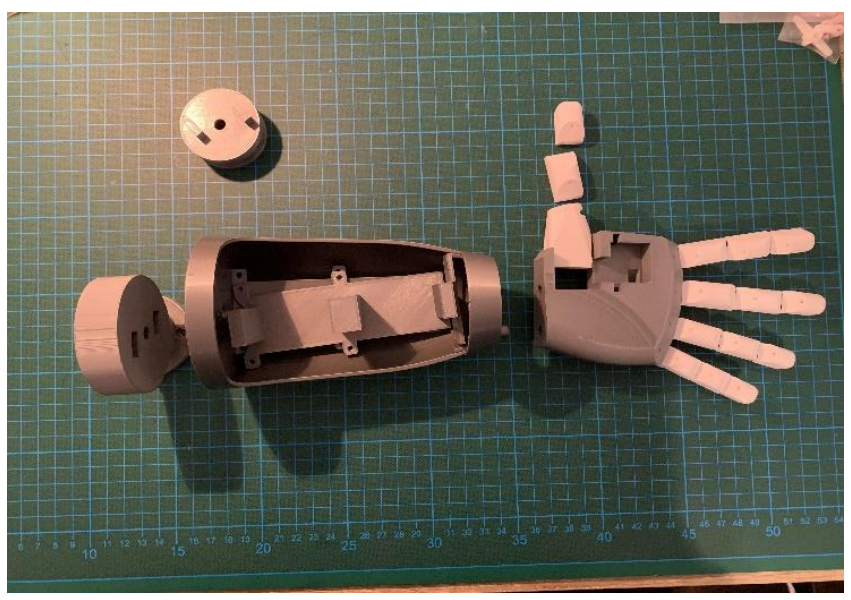

Figure 6. 3D printed parts.

In Figure 7 we can see the servomotor mount, the mount offers the possibility to attach 4 servo motors for controlling the fingers movement. Servo no.1 controls the pinky finger, servo no. 3 controls ring finger, servo no. 2 control the middle finger and servomotor no. 1 controls the index finger and thumb.

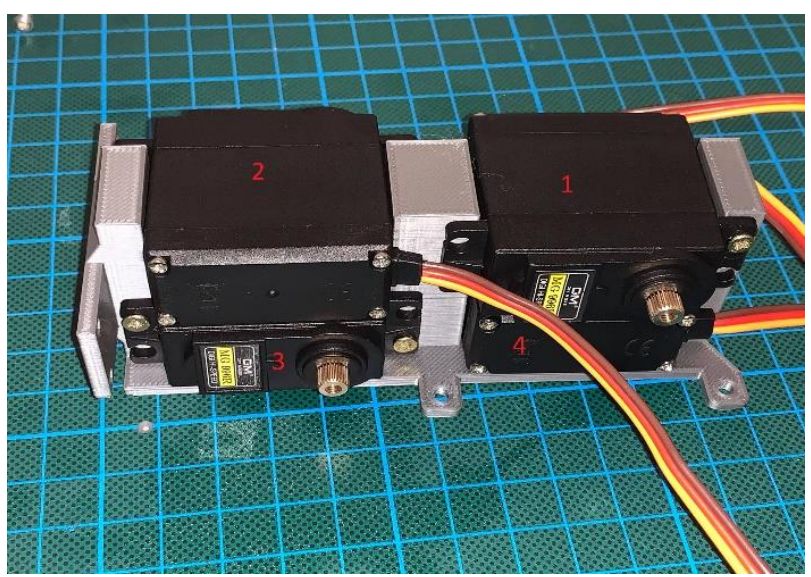

Figure 7. Servomotor mount. 
The movement is done by pulling on a nylon line by a 3D printed wheel (Figure 8), the nylon line is attached to each finger thus reproducing the natural movement of the hand.

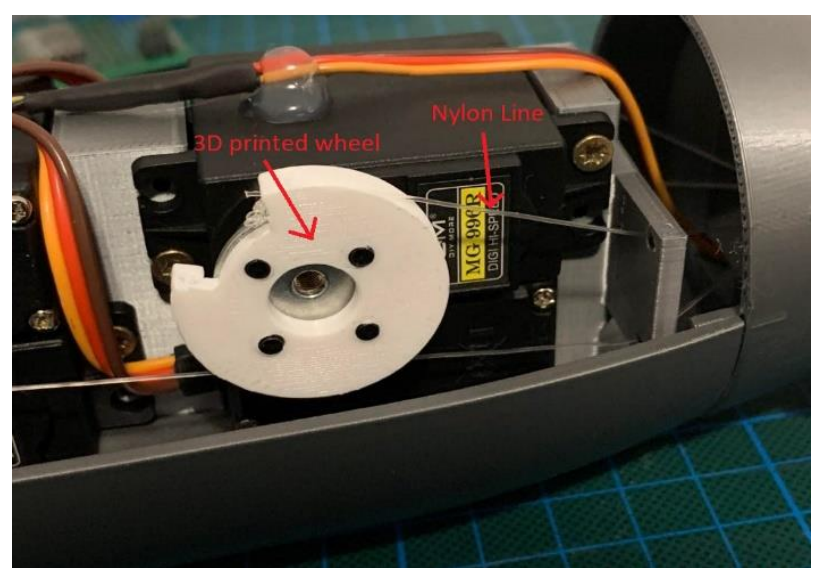

Figure 8. Finger movement.

In Figure 9 we can see the test application that is made in Java programming language, the application has 6 sliders, four for the servomotors that move the fingers, one for the small servomotor that moves the thumb and a extra slider for advanced testing.

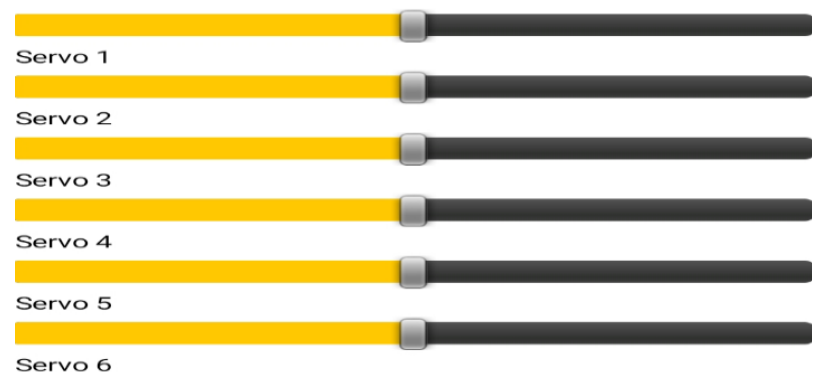

Figure 9. Mobile application.

Each slider sends via the Bluetooth connection a series of numbers, e.g. servo no.1 slider sends numbers in the range of 1000-1180, servo no. 2 send numbers in the range of 2000-2180 etc and in Figure 10 we have the robotic arm fully assembled.

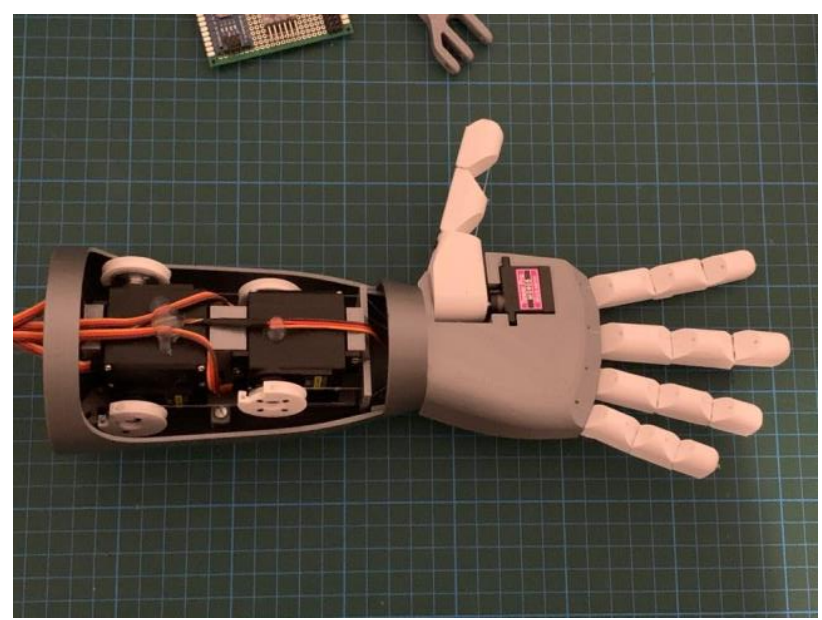

Figure 10. Robotic arm.

\section{CONCLUSIONS}

The process of 3D design/printing is an economical solution for developing prosthesis, the movement mechanism is easy to implement and calibrate. The servomotors used offer the user a stable control of the fingers and the testing application is intuitive and can be programed for other functions.

\section{REFERENCES}

[1] Matt Lombard, Mastering SolidWorks, John Wiley \& Sons, 2018.

[2] Joshua Bloch, Effective Java, Addison-Wesley Professional, 2017.

[3] Douglas Brooks, PCB Currents: How They Flow, How They React (Paperback), Prentice Hall, 2013.

[4] Simon Monk, Programming Arduino: Getting Started with Sketches, McGraw Hill Professional, 2016.

[5] John Boxall, Arduino Workshop: A Hands-on Introduction with 65 Projects, No Starch Press, 2013. 